\title{
Positive polynomial matrices for LPV controller synthesis
}

\author{
Didier Henrion ${ }^{1,2,3}$
}

May 23, 2013

\begin{abstract}
Positive polynomial matrices and linear matrix inequalities (LMI) can be used to design linear parameter varying (LPV) controllers depending polynomially on the scheduling parameters, and robust to polynomial parametric uncertainty. The salient features of the approach are (a) the ability to design a controller of order and structure fixed a priori; (b) the use of a transfer function, or polynomial modeling framework that bypasses difficulties typically encountered with canonical state-space representations of LPV controllers; (c) the existence of a user-friendly Matlab interface to model this class of LMI problems. The main limitation of the approach is the choice of a nominal, or central characteristic polynomial around which the design is carried out.
\end{abstract}

\section{Introduction}

The purpose of this contribution is to survey some achievements of the last decade in the use of polynomial and linear matrix inequality (LMI) methods for designing linear parameter varying (LPV) controllers. No new results are reported here, but various technical statements scattered in the literature are gathered and presented in a hopefully unified fashion. These results are the outcome of eight-year long collaboration (2001-2008) between LAAS-CNRS, an academic research laboratory, and Safran-Snecma, a company dedicated to design, development and production of engines for civil aircrafts, military aircrafts, launch vehicles and satellites. This collaboration resulted in the defense of two $\mathrm{PhD}$ theses $[20,8]$ and several engineering projects and scientific productions, see e.g. $[21,23]$, culminating with the paper [9].

The main objective of the project was the development of computer-aided control system design tools for designing LPV controllers for aircraft turbofan engines. Some of these tools have been integrated into the ATOL framework used by Safran-Snecma to design

\footnotetext{
${ }^{1}$ CNRS; LAAS; 7 avenue du colonel Roche, F-31077 Toulouse; France. henrion@laas.fr

${ }^{2}$ Université de Toulouse; UPS, INSA, INP, ISAE; UT1, UTM, LAAS; F-31077 Toulouse; France

${ }^{3}$ Faculty of Electrical Engineering, Czech Technical University in Prague, Technická 2, CZ-16626 Prague, Czech Republic
} 
new engines and improve control laws of existing engines [23]. The main requisites for controller design were as follows:

- both open-loop plant and controller are given in the form of multi-input multi-output (MIMO) transfer functions, i.e. ratios of numerator and denominator polynomials;

- open-loop plant data (i.e. polynomial coefficients) depend polynomially on the real parametric uncertainty affecting the system; uncertain parameters are confined to a given compact basic semialgebraic set (e.g. a ball, a box);

- controller data (i.e. polynomial coefficients) depend polynomially on the real scheduling parameters; scheduling parameters are confined to a given compact basic semialgebraic set (e.g. a ball, a box);

- controller order and structure are fixed from the outset (e.g. PID controller depending quadratically on two scheduling parameters) independently of the open-loop system order.

In our opinion, the originality of our approach lies in the choice of polynomials and rational transfer functions as modeling objects. This bypasses the standard complications arising from the use of canonical state-space representations in interpolated scheduling control laws and LPV design, see e.g. [24].

The price one has to pay for the ability of designing controllers of fixed complexity is the use of potentially conservative convex linear matrix inequality (LMI) conditions. The main tuning parameter of the approach, which results in a convexification of the design problem, has however the physical interpretation of being a reference, or nominal, or central closed-loop system around which the design is carried out. Mathematically, a convex LMI inner approximation of the nonconvex stability domain is built, in coefficient parameter space, around a so-called central polynomial. Typically, engineering insight yields a reasonable choice of central polynomial around which LPV controller design is achieved.

In this contribution, we outline the whole approach, starting from sufficient LMI conditions for stability of polynomial matrices, and concluding with LMI conditions for robust polynomial LPV controller design. For confidentiality reasons we cannot reproduce the examples of LPV control laws designed for the Safran-Snecma aircraft turbofan engine project.

\section{Stability of polynomial matrices}

The minimum requirement for a feedback control law is closed-loop system stability. Since we follow a polynomial modeling framework, stability amounts to location of the roots of the characteristic polynomial in a specific region of the complex plane. 
We consider regions

$$
\mathcal{S}=\{s \in \mathbb{C}:\left[\begin{array}{l}
1 \\
s
\end{array}\right]^{*} \underbrace{\left[\begin{array}{ll}
s_{11} & s_{21}^{*} \\
s_{21} & s_{22}
\end{array}\right]}_{S}\left[\begin{array}{l}
1 \\
s
\end{array}\right]<0\}
$$

obtained by a conformal mapping of the open unit disk, parametrized by three complex scalars $s_{11}, s_{21}, s_{22}$ gathered into a 2-by-2 Hermitian matrix $S$. As above and throughout the text, the star denotes transpose conjugation. The case $s_{11}=0, s_{21}=1, s_{22}=0$ corresponds to the open left half-plane (continuous-time systems), whereas $s_{11}=-1, s_{21}=$ $0, s_{22}=1$ models the open unit disk (discrete-time systems). We can also consider intersections of several such stability regions, and hence model damping cones or more complicated frequency-domain specifications, see e.g. [11].

A MIMO rational matrix transfer function can be expressed as a left matrix fraction $D^{-1}(s) N(s)$ where $D(s)$ and $N(s)$ are polynomial matrices of the operator $s$ (the Laplace variable for continuous-time systems, the shift variable for discrete-time systems) of appropriate dimensions. In the (generic) absence of pole-zero cancellations, stability of the transfer function amounts to location of the roots of the determinant of $D(s)$ into region $\mathcal{S}$.

Given a polynomial matrix $D(s)=D_{0}+D_{1} s+D_{2} s^{2}+\cdots+D_{d} s^{d}$ of degree $d$, let us denote by

$$
D=\left[\begin{array}{lllll}
D_{0} & D_{1} & D_{2} & \cdots & D_{d}
\end{array}\right]
$$

the constant matrix obtained by appending columnwise matrix coefficients of increasing powers of indeterminate $s$. Let us define

$$
\Pi_{1}=\left[\begin{array}{cccc}
0 & I & & \\
\vdots & & \ddots & \\
0 & & & I
\end{array}\right], \quad \Pi_{2}=\left[\begin{array}{cccc}
I & & & 0 \\
& \ddots & & \vdots \\
& & I & 0
\end{array}\right]
$$

two matrices of size $d n$-by- $(d+1) n$ with $I$ denoting the identify matrix of size $n$. Finally, given a 2-by-2 Hermitian stability matrix $S$ as above, let us define the linear mapping

$$
F(P)=s_{11} \Pi_{1}^{*} P \Pi_{1}+s_{21} \Pi_{2}^{*} P \Pi_{1}+s_{21}^{*} \Pi_{1}^{*} P \Pi_{2}+s_{22} \Pi_{2}^{*} P \Pi_{2}
$$

transforming a Hermitian matrix of size $d n$ into a Hermitian matrix of size $(d+1) n$.

The following theorem gives a necessary and sufficient condition for stability of polynomial matrix $D(s)$, that is, for inclusion of the roots of $\operatorname{det} D(s)$ into region $\mathcal{S}$. The notation $X \succeq 0$ means that $X$ is Hermitian positive semidefinite, i.e. all the eigenvalues of $X$ are real nonnegative.

Theorem 1 Polynomial matrix $D(s)$ is stable if and only if there exists a stable polynomial matrix $C(s)$ and a Hermitian matrix $P$ such that

$$
C^{*} D+D^{*} C-F(P) \succeq 0
$$




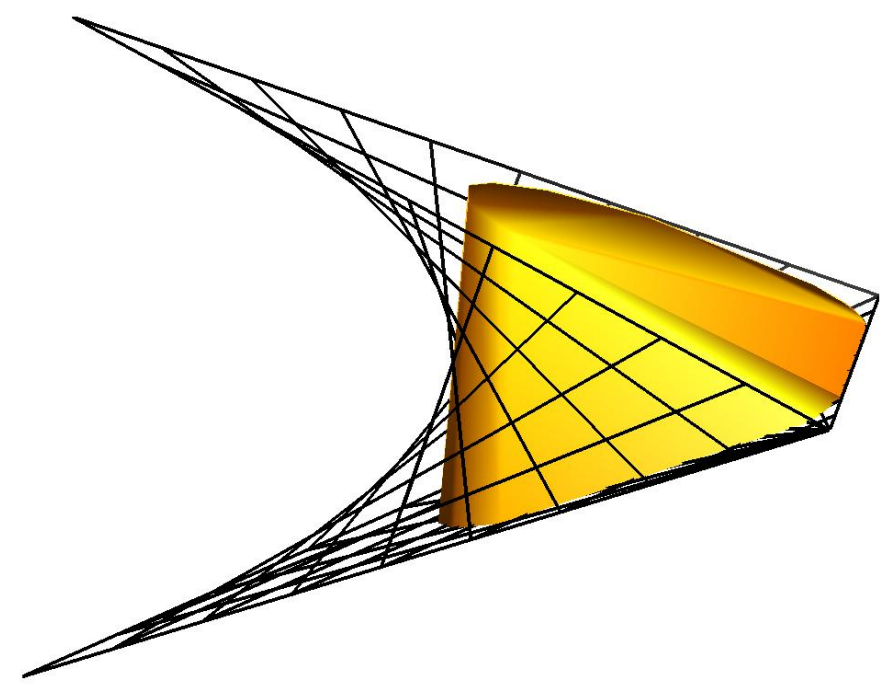

Figure 1: Convex LMI inner approximation of the nonconvex stability region for a thirddegree discrete-time polynomial.

Proof: It can be traced back to [10, 11], see also [13, Lemma 2] and [9, Theorem 1] for alternative proofs. In particular, it must be emphasized that $P$ plays the role of the Hessian matrix of a quadratic Lyapunov function of the linear system with transfer function $D^{-1}(s) C(s)$.

Polynomial matrix $C(s)$ is called the central polynomial. Once it is fixed, inequality (1) becomes linear in $D$ and $P$. This joint linearity is a key characteristic of the LMI conditions of Theorem 1, shared e.g. with the discrete-time stability conditions of [4]. In particular, if $D(s)$ depends linearly on the controller parameters, controller design boils down to solving a convex LMI problem. The whole conservatism of the approach is therefore captured by the choice of central polynomial $C(s)$. Even though this is not developed here, LMI condition (1) can also be used for robustness analysis, see [13, 12].

Geometrically, the condition of Theorem 1 can be interpreted as follows. In the space of polynomial matrix coefficients, a convex region is built around $C$. It is modeled as a projection of an affine section of the cone of positive semidefinite matrices. The operation of projection corresponds to elimination of the variable $P$. This class of convex regions is called semidefinite representable, cf. [1]. It is a very broad class of convex regions. Currently it is not known how versatile it is exactly, but it is conjectured that all convex semialgebraic sets (i.e. convex sets described by polynomial inequalities) can be modelled like that [19], and international research programmes are currently carried out to further investigate these questions at the boarder between convex analysis, real algebraic geometry and mathematical programming.

For illustration, on Figure 1 we represent a convex LMI inner approximation of the nonconvex stability region in the case of scalar $(n=1)$ discrete-time $\left(s_{11}=-1, s_{21}=0\right.$, 
$\left.s_{22}=1\right)$ third degree $(d=3)$ monic $\left(D_{3}=1\right)$ polynomials with real coefficients, for the choice of stable central polynomial $C(s)=s^{3}$. The exact stability domain in the coefficient space $x=\left(D_{0}, D_{1}, D_{2}\right) \in \mathbb{R}^{3}$ is a nonconvex region delimited by two triangles and a parabolic hyperboloid. The LMI approximation (1) is given by

$$
\left\{x \in \mathbb{R}^{3}: \exists p \in \mathbb{R}^{6}:\left[\begin{array}{l}
p_{0} \\
p_{1} p_{3}-p_{0} \\
p_{2} p_{4}-p_{1} p_{5}-p_{3} \\
x_{0} x_{1}-p_{2} x_{2}-p_{4} 2-p_{5}
\end{array}\right] \succeq 0\right\}
$$

where symmetric entries are not reproduced, and vector $p$ of additional variables, or liftings, correspond to the 6 linearly independent entries of the 3-by-3 symmetric matrix $P$.

\section{Fixed-order robust controller design}

Based on Theorem 1, fixed-order robust controller design boils down to convex LMI optimization, and the main tuning parameter, capturing all degrees of freedom as well as all the conservatism of the approach, is the central polynomial. The use of the LMI conditions of Theorem 1 for fixed-order controller design was proposed in [12], and it was used e.g. in $[6,16]$.

\section{1 $\quad H_{\infty}$ performance}

In addition to locating the closed-loop poles into a region $\mathcal{S}$ of complex plane, we may want a control law to ensure some performance requirements. In the case of $H_{\infty}$ performance, we can readily exploit well-known links with robust stability of systems with unstructured uncertainty. We assume now that polynomial matrix is affected by an additive normbounded uncertainty

$$
D_{\delta}(s)=D(s)+\delta N(s), \quad\|\delta\|_{\infty} \leq \gamma^{-1}
$$

where $\delta$ is an unknown real (or complex) valued matrix whose maximum singular value does not exceed a given positive threshold $\gamma^{-1}$. Using the small-gain theorem $[26$, Theorem 9.1], robust stability of matrix $D_{\delta}(s)$ is equivalent to the $H_{\infty}$ performance constraint

$$
\left\|D^{-1}(s) N(s)\right\|_{\infty} \leq \gamma
$$

where the infinity norm denotes the maximum singular value achieved for $s$ along the boundary of open set $\mathcal{S}$.

The following result, proved e.g. in [25, Corollary 1] or [9, Theorem 2], is an extension of Theorem 1 to $H_{\infty}$ performance.

Lemma 1 Given a stable polynomial matrix $C(s)$, rational matrix $D^{-1}(s) N(s)$ is stable with $H_{\infty}$ norm less than or equal to $\gamma$ if there exists a Hermitian matrix $P$ and a real scalar $\lambda$ such that

$$
\left[\begin{array}{cc}
C^{*} D+D^{*} C-F(P)-\lambda C^{*} C & N^{*} \\
N & \lambda \gamma^{2} I
\end{array}\right] \succeq 0
$$


In particular, in $H_{\infty}$ LMI (2) we retrieve the stability LMI (1) in the upper-left block for the choice $\lambda=0$. For examples of lower-order controller design using Lemma 1, see $[14,25]$.

\section{$3.2 \quad H_{2}$ performance}

The extension of Theorem 1 to $H_{2}$ performance was carried out in [8], but since this document is not publicly available, we reproduce the main result below.

If $u$ denotes the input signal, $y$ the output signal, and $G$ the transfer function of a linear plant, the $H_{\infty}$ norm of $G$ can be defined as an energy to energy norm $\|G\|_{\infty}=$ $\sup \|y\|_{2} /\|u\|_{2}$. Similarly, the $H_{2}$ norm can be defined as an energy to peak norm $\|G\|_{2}=$ $\sup \|y\|_{\infty} /\|u\|_{2}$.

Lemma 2 Given a stable polynomial matrix $C(s)$, rational matrix $D^{-1}(s) N(s)$ is stable with $\mathrm{H}_{2}$ norm less than or equal to $\gamma$ if there exists a Hermitian matrix $P$ such that

$$
C^{*} D+D^{*} C-C^{*} C-F(P) \succeq 0, \quad\left[\begin{array}{cc}
P & N^{*} \\
N & \gamma^{2} I
\end{array}\right] \succeq 0
$$

\subsection{Robustness}

For notational simplicity, in stability LMI (1), $H_{\infty}$ LMI (2) or $H_{2}$ LMI (3), let us gather plant (or controller) parameters $N, D$ into a vector $k$.

Let us assume that plant parameters are uncertain, that is, coefficients of polynomial matrices $N(s)$ and $D(s)$ depend on parameters $\delta$ which belong to a given compact set $\Delta$ but which are otherwise unknown. If the dependence of $N$ and $D$ on $\delta$ is polynomial, LMI (1) or (2) or (3) becomes a so-called parametrized, or uncertain LMI

$$
L_{\delta}(k, P) \succeq 0, \quad \forall \delta \in \Delta
$$

which must be solved for $k$ and $P$ uniformly for all possible values of $\delta \in \Delta$. If $\Delta$ is not discrete, then LMI (4) is semi-infinite, in the sense that an infinite number of constraints (one for each value of $\delta$ in $\Delta$ ) must be solved for a finite number of variables (in $k$ and $P)$. Such robust LMIs are extensively studied in [2] and [22].

In many cases of interest, an equivalent robust LMI condition

$$
L(k, P) \succeq 0
$$

can be derived to remove dependence on uncertain parameter $\delta$, in the sense that there is a solution to uncertain LMI (4) if and only if there is a solution to robust LMI (5). Depending on the class of uncertainty set $\Delta$, robust LMI (5) can be significantly larger than uncertain LMI (4), but this is the price to pay to convert a semi-infinite LMI into a finite LMI. The simplest example is when $N$ and $D$ depend affinely on $\delta$ and $\Delta$ is a polytope, the so-called polytopic uncertain model [7,3]. Robust LMI (5) then corresponds to all the instances of uncertain LMI (4) at vertices of $\Delta$. In the next section, in the context of LPV controller design, we describe a general technique to deal with parametrized LMIs when the dependence on the parameters is polynomial. 


\section{LPV controller design}

In this section we assume that numerator and denominator polynomial matrices now depend on a vector of parameters

$$
\begin{aligned}
& N(s, \theta)=N_{0}(\theta)+N_{1}(\theta) s+N_{2}(\theta) s^{2}+\cdots+N_{d}(\theta) s^{d} \\
& D(s, \theta)=D_{0}(\theta)+D_{1}(\theta) s+D_{2}(\theta) s^{2}+\cdots+D_{d}(\theta) s^{d}
\end{aligned}
$$

which is assumed to belong to a given set

$$
\theta \in \Theta
$$

Such a parameter depends on the operation condition of the open-loop system, and it can be measured on-line by the controller. For simplicity, we assume first that parameter $\theta$ does not vary in time, even though at the end of the section we will indicate how to relax this assumption. In this context, the objective of linear parameter varying (LPV) controller design is to find a controller depending explicitly on $\theta$ and that ensures closedloop stability and performance.

\subsection{A hierarchy of LMI problems}

Following the approach described in the previous section, the designer comes up with a parametrized LMI

$$
L_{\theta}(k, P) \succeq 0, \quad \forall \theta \in \Theta
$$

to be solved for controller coefficients $k$ and Lyapunov coefficients $P$, where $L_{\theta}$ is one of the LMI mapping (1), (2) or (3) with an additional explicit dependence of $N$ and $D$ on $\theta$.

If the dependence on $\theta$ is polynomial, i.e.

$$
\begin{aligned}
& N(s, \theta)=\sum_{\alpha \in \mathbb{N}^{q}} N_{\alpha}(s) \theta^{\alpha} \\
& D(s, \theta)=\sum_{\alpha \in \mathbb{N}^{q}} D_{\alpha}(s) \theta^{\alpha}
\end{aligned}
$$

where in the above sums $\alpha \in \mathbb{N}^{q}$ is a vector of indices, $\theta^{\alpha}=\theta_{1}^{\alpha_{1}} \theta_{2}^{\alpha_{2}} \cdots \theta_{q}^{\alpha_{q}}$, and if set $\Theta$ is basic semi-algebraic, i.e. if it is modeled as

$$
\Theta=\left\{\theta \in \mathbb{R}^{q}: g_{i}(\theta) \geq 0, i=1, \ldots, r\right\}
$$

for explicitly given polynomials $g_{i}(\theta)$, then we can use recent results on positive polynomials [17] to solve parametrized LMI (6) via a hierarchy of robust LMIs.

In particular, we can use Putinar's Positivstellensatz under the non-restrictive assumption that the semialgebraic set $\Theta$ is compact and that it is included in a ball of radius $\rho$ centered around the origin. For example, this is ensured if $g_{i}(\theta)=\rho^{2}-\theta^{*} \theta$ for some $i$.

Lemma 3 There exists $k$ and $P$ such that $L_{\theta}(k, P) \succ 0$ for all $\theta \in \Theta$ if and only if there exists sum-of-squares matrix polynomials $M_{i}(\theta)$ such that $L_{\theta}(k, P)=M_{0}(\theta)+$ $\sum_{i=1}^{r} g_{i}(\theta) M_{i}(\theta)$. 
Proof: See [22, Theorem 24] and [17, Theorem 2.22], an extension to the matrix case of scalar result by Putinar used for polynomial optimization.

Practically speaking, the discrepancy between the strict inequality in Lemma 3 and the non-strict inequality in (6) is not relevant. In terms of implementation, the constraint that $L_{\theta}(k, P)$ is a linear combination of sum-of-squares (SOS) matrix polynomials can be expressed as an LMI, as soon as the degree of the SOS multipliers is fixed. The decision variables are then $k$ and $P$, but also the Gram matrices of the SOS multipliers, see [17, Chapter 2]. By increasing the degree of the SOS multipliers, we obtain a hierarchy of robust LMI problems of increasing size whose solution is guaranteed to be equivalent, asymptotically, to the solution of the polynomially parametrized LMI (6). The Matlab modeling environment YALMIP [18] allows to model matrix SOS problems and generate the corresponding LMI problems in a user-friendly way. The LMI problems can then be solved by any semidefinite programming solver (e.g. SeDuMi, PENSDP, SDPT3, SDPA, CSDP).

\subsection{Parameter-dependent Lyapunov functions}

So far we have considered a polynomially parametrized LMI (6) which is an instance of stabilization LMI (1), $H_{\infty}$ LMI (2) or $H_{2}$ LMI (3) in which decision variable $P$ is the Hessian matrix of a Lyapunov function certifying stability and performance. The Lyapunov function does not depend on scheduling parameter $\theta$, hence we are assessing quadratic stability of the LPV system, i.e. stability for all possible time variations of $\theta$, including jumps or discontinuities. If we have some information of the time variation of $\theta$ (e.g. bounds on the rate of variation), or if $\theta$ is assumed to be constant, but otherwise unknown, this information can be incorporated in parametrized LMI (6) by allowing Lyapunov matrix $P$ to depend on $\theta$. We can use the same robust LMI as in Lemma 3 as soon as the dependence of $P$ on $\theta$ is polynomial. The trade-off to be found is between the complexity (degree) of the dependence of $P$ on $\theta$ and the size of LMI problems in the hierarchy.

Finally, we can additionally assume as in paragraph 3.3 that the open-loop plant parameters are uncertain, depending polynomially on a vector or matrix $\delta$ of uncertain parameters assumed to belong to a compact basic semialgebraic set $\Delta$. We can use the same hierarchy of LMI problems as in Lemma 3, the only difference being that the controller is not allowed to depend polynomially on $\delta$ (uncertain parameters are not measurable). In contrast, Lyapunov matrix $P$ can depend simultaneously on scheduling parameter $\theta$ and uncertain parameter $\delta$.

\section{Conclusion}

We have described in a unified framework the application of recent positive polynomial and LMI techniques to the design of fixed-order robust LPV controllers for linear systems described by input-output transfer functions whose coefficients depend polynomially on scheduling parameters and uncertain parameters. The main tuning parameter of the 
approach is the central polynomial, a reference closed-loop system around which design is carried out. Once the central polynomial is fixed, design boils down to solving a hierarchy of LMI problems. These problems can be modeled easily under Matlab with the YALMIP interface, and they can be solved with any semidefinite programming solver. These techniques have been succesfully applied within the scope of a eight-year industrial project with the French aerospace engine manufacturer Safran-Snecma. Outcomes have been the defense of two $\mathrm{PhD}$ theses $[20,8]$ and an industrial software environment to assist engineers when developing advanced control laws for new aircraft engine prototypes [23].

\section{Acknowledgments}

This work resulted from a collaboration with Jacques Bernussou, David Boyer, Wilfried Gilbert, Luc Reberga and Florian Vary. It was funded by research contracts between LAAS-CNRS and the Safran-Snecma company. The research of Didier Henrion on positive polynomials was also funded in part by projects nos. 102/06/0652, 102/08/0186, 103/10/0628 of the Grant Agency of the Czech Republic, and programme no. MSM6840770038 of the Ministry of Education of the Czech Republic. I am grateful to Arash Sadeghzadeh for pointing out a mistake in the original formulation of condition (3).

\section{References}

[1] A. Ben-Tal, A. Nemirovski. Lectures on modern convex optimization. SIAM, Philadelphia, 2001.

[2] A. Ben-Tal, A. Nemirovski, L. El Ghaoui. Robustness. In H. Wolkowicz, R. Saigal, L. Vandenberghe (Editors). Handbook of Semidefinite Programming, Kluwer Academic Publishers, Dordrecht, 2000.

[3] S. Boyd, L. El Ghaoui, E. Feron, V. Balakrishnan. Linear matrix inequalities in system and control Theory. SIAM, Philadelphia, 1994.

[4] M. C. de Oliviera, J. Bernussou, J. C. Geromel. A new discrete-time robust stability condition. Syst. Control Lett., 37(4):261-265, 1999.

[5] J. C. Doyle, B. A. Francis, A. R. Tannenbaum. Feedback control theory. MacMillan, New York, 1992.

[6] B. Dumitrescu. Positive trigonometric polynomials and signal processing applications. Springer, Berlin, 2007.

[7] J. C. Geromel, P. L. D. Peres, J. Bernussou. On a convex parameter space method for linear control design of uncertain systems. SIAM J. Control Optim., 29:381-402, 1991. 
[8] W. Gilbert. Synthèse LPV polynomiale appliquée à la commande de turboréacteurs. PhD thesis, INSA Toulouse, LAAS-CNRS and Safran-Snecma (not publicly available), Nov. 2008.

[9] W. Gilbert, D. Henrion, J. Bernussou, D. Boyer. Polynomial LPV synthesis applied to turbofan engines. Control Eng. Practice, 18:1077-1083, 2010.

[10] D. Henrion, D. Arzelier, D. Peaucelle, M. Šebek. An LMI condition for robust stability of polynomial matrix polytopes. Automatica, 37(3):461-468, 2001.

[11] D. Henrion, O. Bachelier, M. Šebek. D-stability of polynomial matrices. Int. J. Control, 74(8):845-856, 2001.

[12] D. Henrion, M. Šebek, V. Kučera. Positive polynomials and robust stabilization with fixed-order controllers. IEEE Trans. Autom. Control, 48(7):1178-1186, 2003.

[13] D. Henrion, D. Arzelier, D. Peaucelle. Positive polynomial matrices and improved LMI robustness conditions. Automatica, 39:1479-1485, 2003.

[14] D. Henrion, P. Ghildiyal. Optimization with polynomials and fixed-order robust controllers: a design example. Views-and-News letter of the SIAM Activity Group on Optimization, 15(2):15-17, 2004

[15] D. Henrion, J.-B. Lasserre. Convergent relaxations of polynomial matrix inequalities and static output feedback. IEEE Trans. Autom. Control, 51(2):192-202, 2006.

[16] A. Karimi, H. Khatibi, R. Longchamp. Robust control of polytopic systems by convex optimization. Automatica 43(8):1395-1402, 2007.

[17] J.-B. Lasserre. Moments, positive polynomials and their applications. Imperial College Press, London, UK, 2009.

[18] J. Löfberg. YALMIP: a toolbox for modeling and optimization in Matlab. Proc. IEEE CACSD Symposium, Taipei, Taiwan, 2004.

[19] A. Nemirovski. Advances in convex optimization: conic programming. Proc. Int. Congress Math., Madrid, August 2006.

[20] L. Reberga. Commande robuste multivariable des turboréacteurs. PhD thesis, INSA Toulouse, LAAS-CNRS and Safran-Snecma (not publicly available), Sep. 2005.

[21] L. Reberga, D. Henrion, J. Bernussou, F. Vary. LPV modeling of a turbofan engine. Proc. IFAC World Congress on Automatic Control, Prague, Czech Rep., July 2005.

[22] C. W. Scherer. LMI relaxations in robust control. Europ. J. Control, 12(1):3-29, 2006.

[23] F. Vary, L. Reberga. Programming and computing tools for jet engine control design. Proc. IFAC World Congress on Automatic Control, Prague, Czech Rep., July 2005.

[24] M. G. Wassink, M. van de Wal, C. W. Scherer, O. Bosgra. LPV control for a wafer stage: beyond the theoretical solution Control Eng. Practice, 13(2):231-245, 2005. 
[25] F. Yang, M. Gani, D. Henrion. Fixed-order robust H-infinity controller design with regional pole assignment. IEEE Trans. Autom. Control, 52(10):1959-1963, 2007.

[26] K. Zhou, J. C. Doyle, K. Glover. Robust and optimal control. Prentice-Hall, Upper Saddle River, 1996. 